\title{
A DECISION TREE BASED PEDOMETER AND ITS IMPLEMENTATION ON THE ANDROID PLATFORM
}

\author{
Juanying Lin, Leanne Chan and Hong Yan \\ Department of Electronic Engineering, \\ City University of Hong Kong, Hong Kong, China \\ juanyilin2-c@my. cityu.edu.hk, \\ leanne.chan@cityu.edu.hk, h.yan@cityu.edu.hk
}

\begin{abstract}
This paper describes a decision tree (DT) based pedometer algorithm and its implementation on Android. The DT- based pedometer can classify 3 gait patterns, including walking on level ground (WLG), up stairs (WUS) and down stairs (WDS). It can discard irrelevant motion and count user's steps accurately. The overall classification accuracy is $89.4 \%$. Accelerometer, gyroscope and magnetic field sensors are used in the device. When user puts his/her smart phone into the pocket, the pedometer can automatically count steps of different gait patterns. Two methods are tested to map the acceleration from mobile phone's reference frame to the direction of gravity. Two significant features are employed to classify different gait patterns.
\end{abstract}

\section{KEYWORDS}

Pedometer, Decision Tree, Sensor, Gait analysis \&Classification, Mobile Phone Applications.

\section{INTRODUCTION}

Commonly used pedometers are often built as separate products and their accuracy is often affected by random motions. In this paper, we present a new method to count steps of walking using a mobile phone. We use several sensors to extract signal features and a decision tree to perform data classification. Gyroscopes and accelerometers are wildly used to detect human motions. Gyroscope sensor is used to measure the angular velocity of an object. Doheny et al. used a single gyroscope to analyze spatial gait [1]. Lim et al. proposed a gyroscope-based pedometer [2]. A gyroscope is adhered to the right shank segment to detect user's motion. The work presented here uses gyroscope to measure angular velocity of user's thigh, when the phone is in the user's pocket as shown in Figure 1.

The accelerometer can be used as a sensor to measure the acceleration of an object. Aguiar et al. used the accelerometer embedded in smart phone to detect falling of the elder [3]. Mantyjarvi et al. used accelerometers to recognize human motions [4]. The magnetic field sensor is often used in global positioning system navigation. In this work, data from this sensor are used to generate a rotation matrix. Using the matrix and the original acceleration, the vertical acceleration can be determined. Decision tree is one of the predictive modeling approaches used in statistics, data mining and machine learning. In a decision tree [5], leaves represent target values, which are also called class labels, and branches represent measurements about an item, which is also called a feature. Manap et al. used decision tree to detect parkinsonian gait motor impairment [6].

David C. Wyld et al. (Eds) : CCSIT, SIPP, AISC, NLP - 2015

pp. 73-83, 2015. (C) CS \& IT-CSCP 2015

DOI : $10.5121 /$ csit.2015.50407 


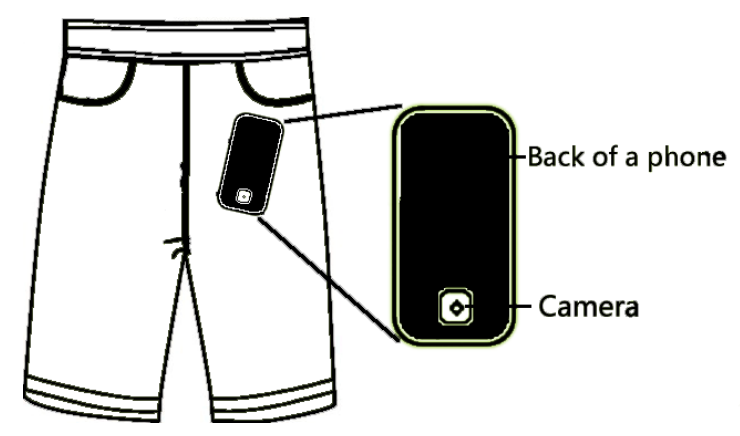

Figure 1. An example of a mobile phone in the user's pocket.

In the work by Lovell et al. [7], a sliding window with a size of 128 samples is used to segment signal of acceleration. In our work, an angular velocity based algorithm is developed to segment the signal of acceleration. Using this algorithm, classification and step counting can be done at the same time. In many pervious work of pedometer or gait classification, such as [2], [7], [8], researchers did not consider the capacity of anti-interference of their systems. In our method, daily irrelevant motions are added to the training set to improve the capacity of anti-interference of the system.

\section{SYSTEM STRUCTURE}

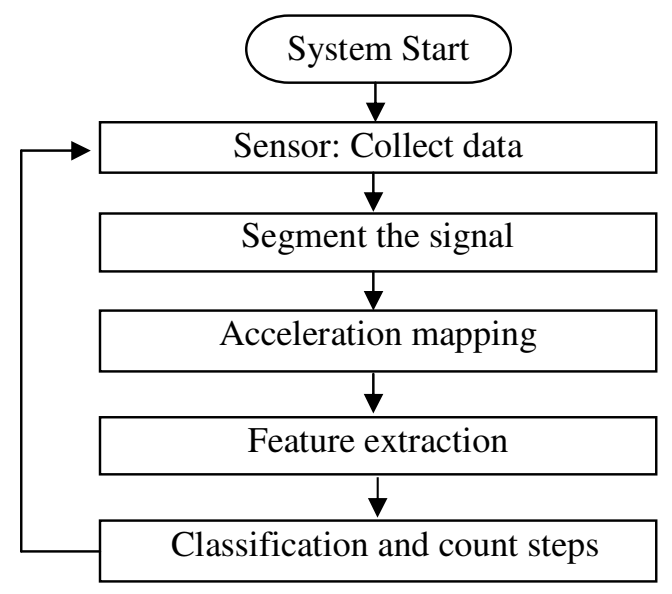

Figure 2. System follow chart of the pedometer.

The system structure of the proposed pedometer is shown in Figure 2. Signals of original acceleration, angular velocity and magnetic field are recorded with a sampling frequency of 100 $\mathrm{Hz}$. Then the signals will be cut into small segments. After that, original acceleration is mapped to the direction of the gravity. The features are extracted from each segment. Finally, all features are sent to the decision tree to classify each segment. 


\section{Signal Processing Algorithms}

\subsection{Algorithms for signal segmenting}

To the thigh, a cycle of walking only contains 2 phases, forward rotation (FR) and backward rotation (BR). According to the case shown in Figure 1 and the reference frame of gyroscope shown in Figure 3, BR and FR can be detected by the x-axis of gyroscope in mobile phone.

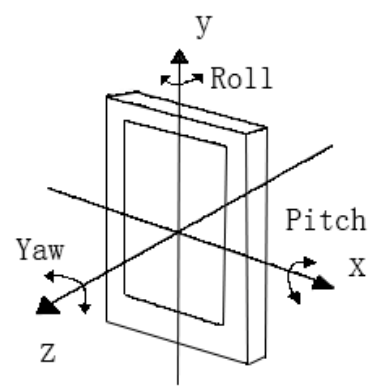

Figure 3. Three axes of the gyroscope in a mobile phone.

Most mobile phones now have a large screen and occupy most space of user's pocket. Therefore, the position of mobile phone is usually stable in the user's pocket and it is reliable to use the Xaxis to detect FR and BR. FR and BR can be easily recognized in the signal of $x$-axis as shown in Figures 5, 6 and 7. The principle of the algorithm is to detect FR of user's thigh and use it to separate signal of each step. The system will continue to monitor the angular velocity of $\mathrm{x}$-axis and detect FR.

If there are 15 consecutive data points whose values are all less than $-1 \mathrm{rad} / \mathrm{s}$, an FR is detected. The start point of a segment is the first data point with positive value after the FR. The start point is located by monitoring the first positive point after detecting the 15 consecutive negative points. The end point of a segment is the last peak whose value is larger than $1 \mathrm{rad} / \mathrm{s}$, before the FR of the next step. A peak is located by checking whether there is a data point denoted by $x(n)$ that meets the requirement : $x(n)-x(n-1)>0$ and $x(n+1)-x(n)<0$, where $\mathrm{n}$ denotes the index of the data point. After setting the start point, if no FR is detected, the end point of the segment will be set to be the 150 th data point after the start point. The signals of angular velocity and vertical vibration are segmented according to start points and end points as shown in Figures 4, 5, 6 and 7. FR is an important element of a walk-like event. If no FR is detected, no segments will be created as illustrated by the signals after the segmentation in Figure 8. Therefore, some irrelevant motions are discarded and the reliability of the system is improved.

Using this algorithm, one segment represents a walk-like event. The system can simply count the number of segments, which are considered to be true walk events by a decision tree, and obtain the number of steps of different gait patterns as shown in Figure 8. Let $S_{\mathrm{WLG}}$, $S_{\mathrm{WUS}}$, and $S_{\mathrm{WDS}}$ denote the number of steps of the 3 gait patterns. The mobile phone only monitors one of user's thighs. Therefore, one segment represents 2 steps.

If a sliding window is used, one step might be detected with 2 consecutive windows, and the number of segments is not equal to the number of steps. Also, a step near the boundary of a segment might be missed, if the step counting algorithm is applied to a larger segment that consists of several small segments. 


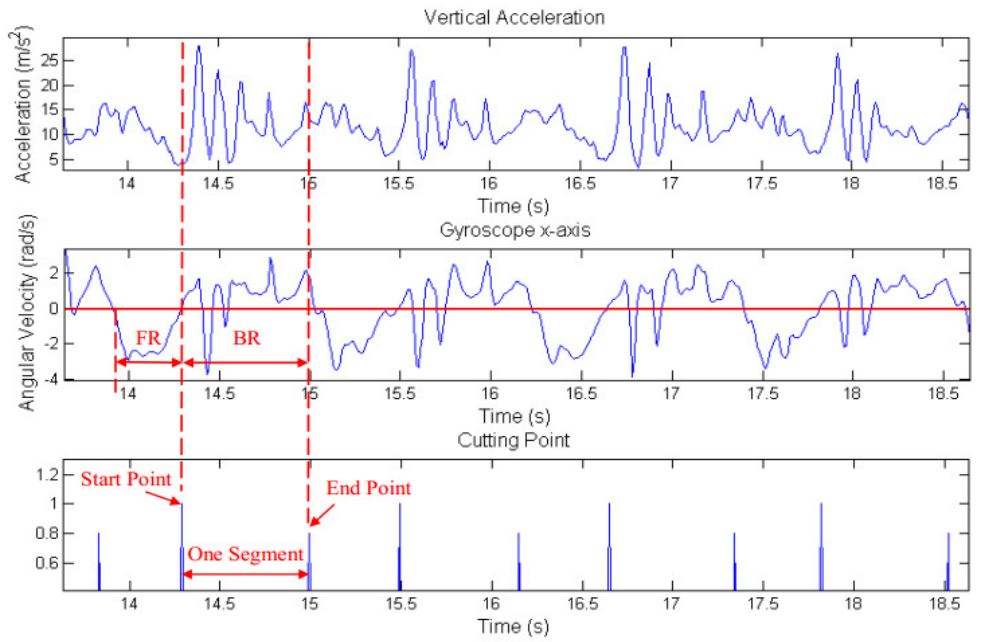

Figure 4. Signals of walking on a level ground

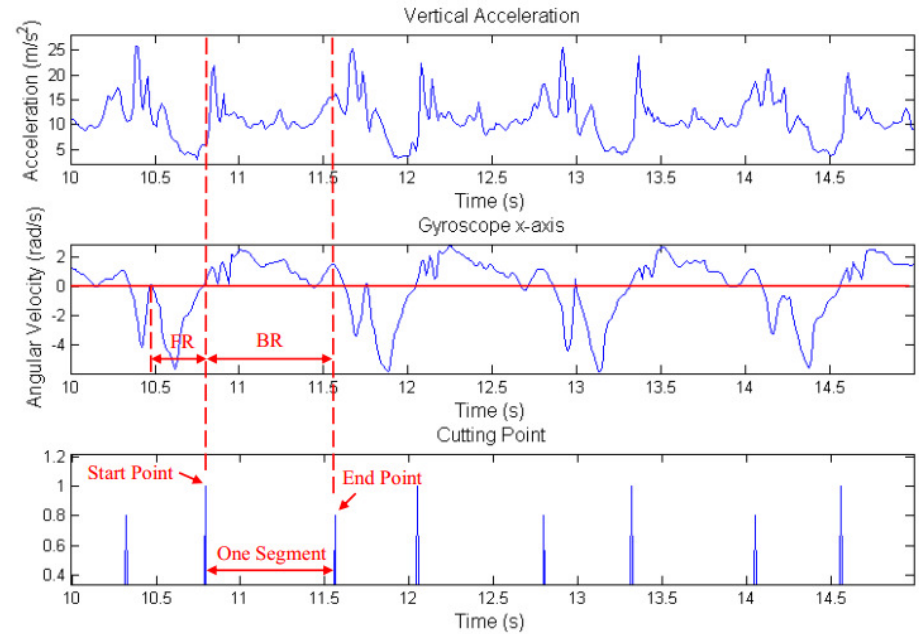

Figure 5. Signals of walking up stairs.

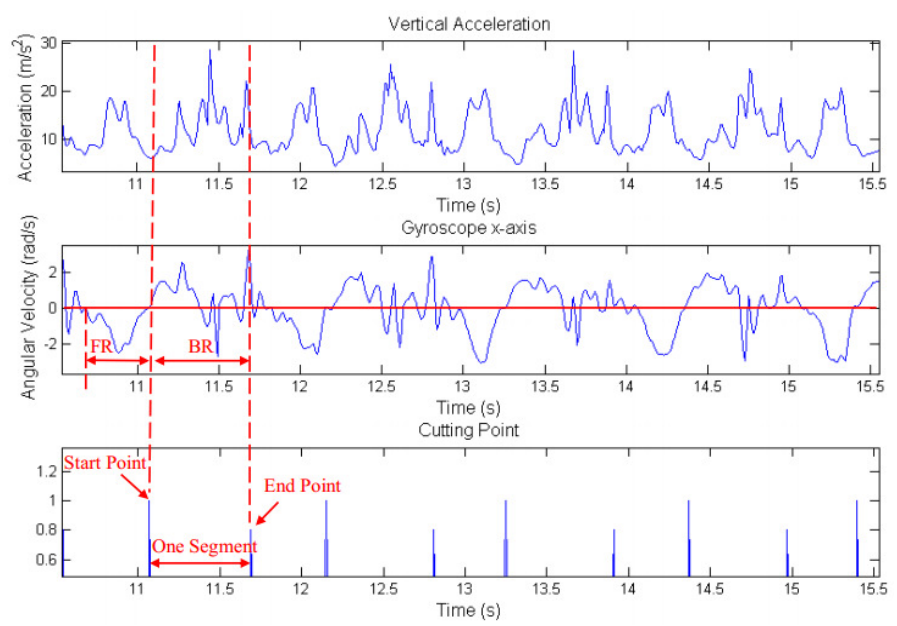

Figure 6. Signals of walking down stairs. 


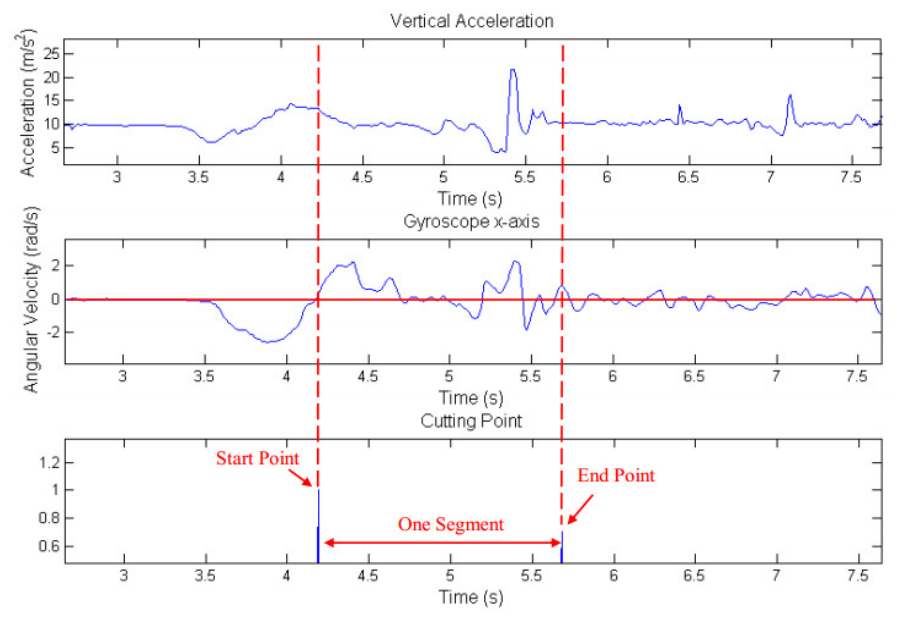

Figure 7. Signals of walking down stairs.

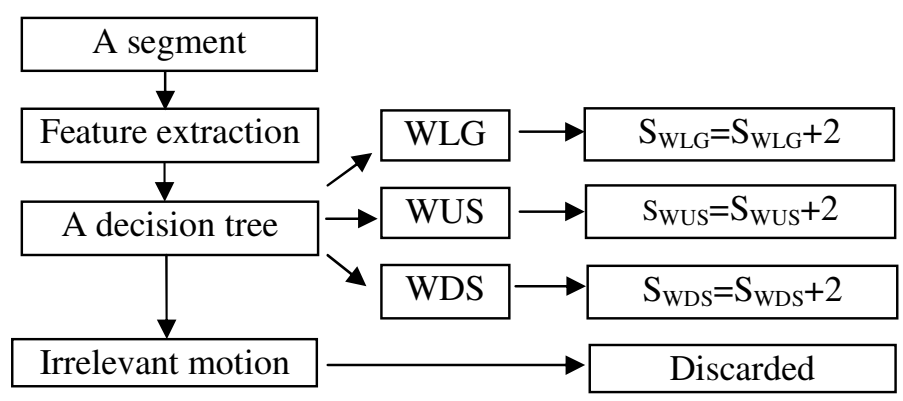

Figure 8. Gait classification and step counting.

\subsection{Acceleration mapping}

The acceleration given by the mobile phone is respected in the mobile phone reference frame shown in Figure 3. Vertical vibration is a significant signal induced by the walk. Therefore, original acceleration needs to be mapped to the direction of the gravity to generate the signal of vertical vibration. There are two methods to achieve it.

In the first method, we calculate the angle between vector of linear acceleration provided by linear acceleration sensor and the vector of $g$ provided by gravity sensor, where $g$ denotes the acceleration due to gravity, and $|\mathrm{g}|=9.8 \mathrm{~m} / \mathrm{s}^{2}$. Let $\mathrm{A}_{\text {linear }}$ denote the linear acceleration, $\mathrm{A}_{\mathrm{GD}}$ denote the value of the acceleration in the direction of gravity, and $\mathrm{x}_{\text {linear }}, \mathrm{y}_{\text {linear }}$ and $\mathrm{z}_{\text {linear }}$ denote elements of the vector of $\mathrm{A}_{\text {linear }}$ respectively, then they can be computed as follows.

$$
\left|\vec{A}_{\text {linear }}\right|=\sqrt{x_{\text {linear }}^{2}+y_{\text {linear }}^{2}+z_{\text {linear }}^{2}}
$$




$$
\begin{aligned}
& \cos \left\langle\vec{A}_{\text {linear }} \cdot \vec{g}\right\rangle=\frac{\vec{A}_{\text {imear }} \cdot \vec{g}}{\left|\vec{A}_{\text {linear }}\right||\vec{g}|} \\
& A_{G D}=-\cos \left\langle\vec{A}_{\text {linear }} \cdot \vec{g}\right\rangle \cdot\left|\vec{A}_{\text {inear }}\right|
\end{aligned}
$$

In the second method, a rotation matrix can be generated by getRotationMatrix, a function provided by Android, using data from the accelerometer and the magnetic field. Then the original acceleration can be mapped to the direction of gravity. Let $\mathrm{A}_{\mathrm{GD}}$ denote the value of the acceleration in the direction of gravity, $\mathrm{M}_{\text {rotation }}$ denote the rotation matrix and $\mathrm{A}_{\text {original }}$ denote the vector of acceleration respecting to mobile phone's reference frame, then

$$
\left[0,0, A_{G D}\right]=M_{\text {rotation }} \times A_{\text {original }}
$$

In Android, there are two kinds of sensors, hardware-based sensors and software-based sensors. Software-based sensors need data from several hardware-based sensors to produce its own data [9]. This means they need more time to process their data. Linear acceleration sensor and gravity sensor are software-based. Method 2 is better than Method 1 because the former is based on hardware-based sensors and needs less time to generate the data.

\section{SIGNIFICANT FEATURES}

In Figure 9, $\mathrm{D}_{\mathrm{FS}}$ denotes the distance between one's foot and the surface of ground or stairs. Length $_{M}$ denotes the length between the start point and the point of the maximum in a segment. Length $_{S}$ denotes the length of a segment. In the signal of vertical vibration, two significant features, location of peak in a segment and variance are found to classify different gait patterns.

\subsection{Location of the maximum in a segment}

The definition is shown as follows. Location $_{M}$ denotes the location of the maximum in a segment.

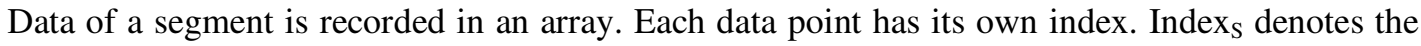

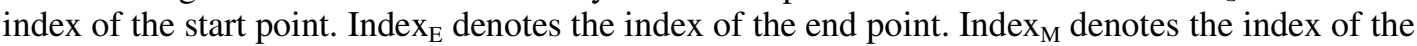

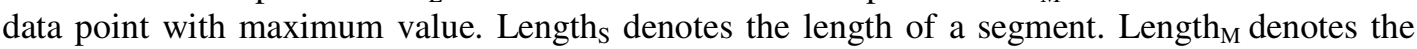
distance between the start point and the point with the maximum value.

$$
\begin{aligned}
& \text { Length }_{M}=\text { Index }_{M}-\text { Index }_{S} \\
& \text { Length }_{S}=\text { Index }_{E}-\text { Index }_{S} \\
& \text { Location }_{\mathrm{M}}=\frac{\text { Length }_{\mathrm{M}}}{\text { Length }_{\mathrm{S}}} \times 100
\end{aligned}
$$




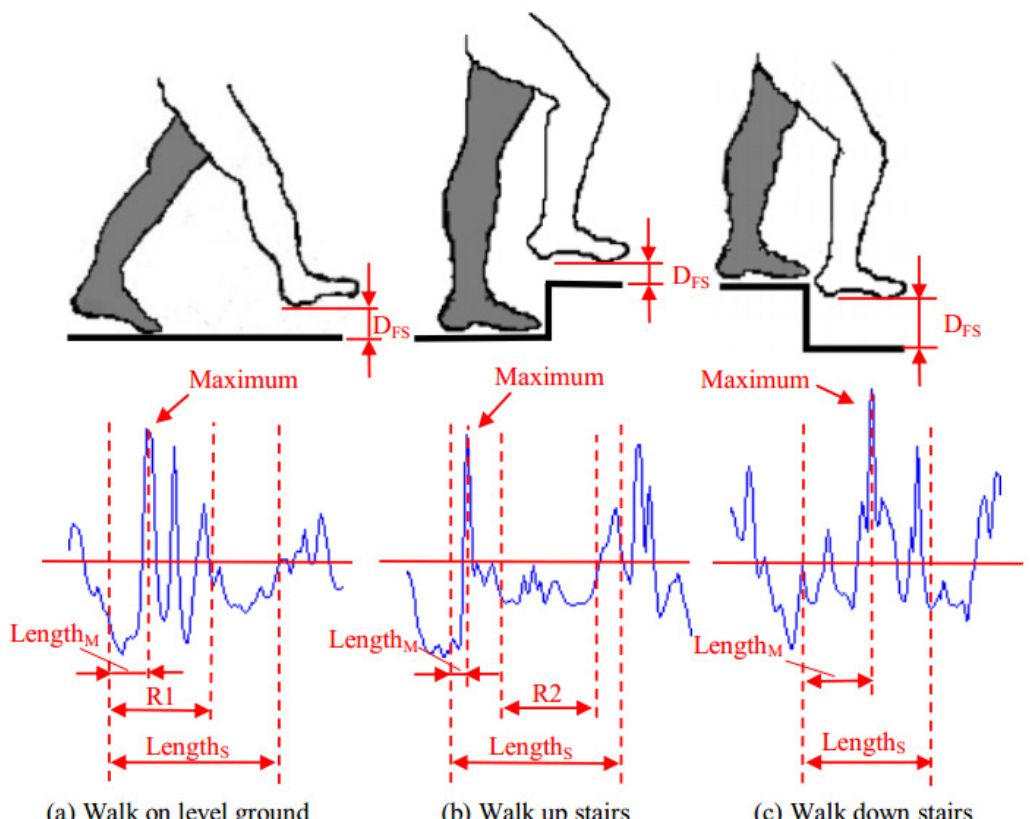

(a) Walk on level ground

(b) Walk up stairs

(c) Walk down stairs

Figure 9. Gait analysis and signal of vertical vibration

Signal in a segment represents the BR of user's thigh, as shown in Figure 9. In motions of walking on level ground or walking up stairs, when the user begins to rotate his/her thigh backward, his/her foot will soon touch the surface, due to small $\mathrm{D}_{\mathrm{FS}}$. Then vibration is induced by heel strike. Therefore, the maximum is located near the start point of a segment. When walking down stairs, $\mathrm{D}_{\mathrm{FS}}$ is larger. After beginning to rotate one's thigh backward, one's foot will not soon touch the surface and induce vibration. Then the maximum is not located near the start point. This feature can separate the motion of walking down stairs from the other 2 gait patterns.

\subsection{Variance of a segment}

Let $\mathrm{D}_{\mathrm{a}}$ denote the average of data values in a segment, $\mathrm{n}$ denote the number of data points, and $\mathrm{Di}$ denote a specified data point. Var denotes the variance of a segment. We calculate the variance of a segment as follows

$$
\begin{aligned}
D_{a} & =\frac{1}{n} \sum_{i=1}^{n} D_{i} \\
V a r & =\frac{1}{n} \sum_{i=1}^{n}\left(D_{i}-D_{a}\right)^{2}
\end{aligned}
$$

In Figure 9, R1 and R2 denote 2 ranges in the signals of walking on level ground and down stairs respectively. R1 is a range of vibration. This range will increase the variance of the segment. Compared with $\mathrm{R} 1, \mathrm{R} 2$ is a range that is relatively flat. $\mathrm{R} 2$ will decrease the variance of the segment. Therefore, the variance of a segment of walking on level ground will be larger than that in a segment of walking up stairs. Using this feature, the motion of walking on level ground can be distinguished from the motion of walking up stairs. 


\section{TRAINING SET AND DECISION TREE}

Five subjects, participate in an experiment to create a training set with 4 kinds of motions, including the 3 gait patterns and irrelevant motion. We choose the decision tree as the classification engine since it has a very low computational complexity and can be implemented on a mobile computing unit (MCU) efficiently [10-12]. In order to avoid imbalanced distribution of different classes in a decision tree, the amount of each class in a training set should be balanced. If one class is the majority in a training set, the decision tree created by this training set is more likely to classify an unknown instance to that class. Then C4.5 algorithm in Weka is used to identify distinct features and create a decision tree, according to the training set. Six features are selected to create the decision tree as shown in Figure 10. In this figure, "Ground" denotes walking on level ground. "Up" denotes walking up stairs. "Down" denotes walking down stairs. "Other" denotes irrelevant motion. In the signal of vertical acceleration, AcceleGMin denotes the minimum value, AcceleGMax denotes the maximum value, AcceleGAverage denotes the average, AcceleGMaxL denotes the location of the maximum and AcceleGVar denotes the variance. In the signal of angular velocity, GyroMin denotes the minimum.

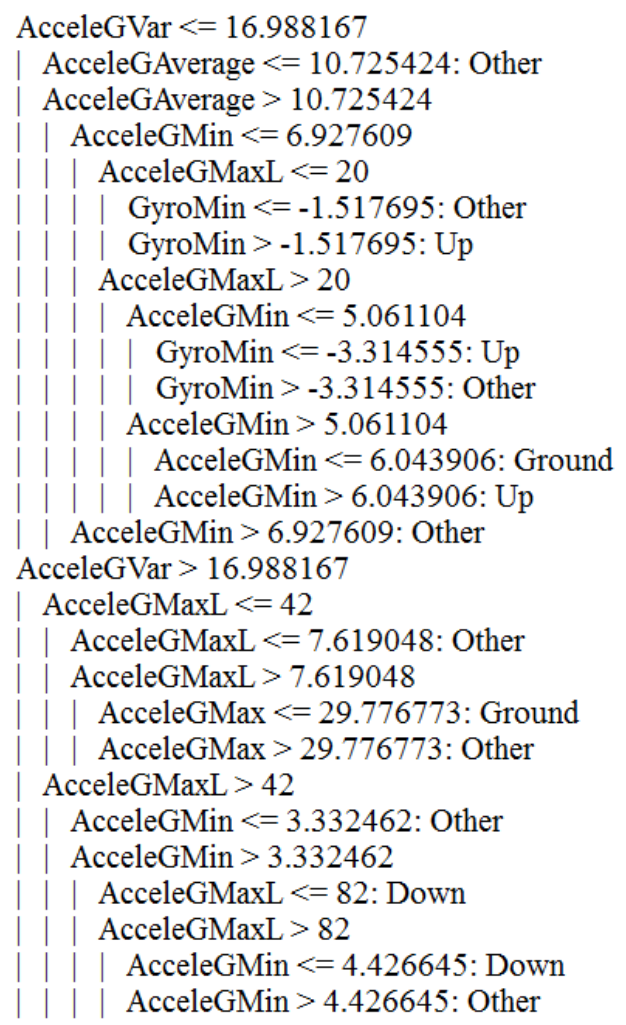

Figure 10. Decision tree used in the system

While creating the classifier Weka also evaluate the performance of this predictive model. Cross validation is a common method to evaluate the accuracy of classifiers [10]. In Leave One-Out (LOO) cross validation, one subject is used for testing and the rest are used for training. The classification result is then computed and repeated until all subjects have participated in the testing dataset. The overall classification result is then computed as the average of all testing subjects [13]. Here 10-folder cross-validation is used to measure the accuracy of this classifier. In 
the cross-validation, the whole training dataset is divided into 10 subsets. One subset is used for testing the rest are used for training. The classification accuracy of this decision tree is $92.3645 \%$. Another measure of classification algorithms performance is a confusion matrix [14]. Precision and recall are typical classification performance measures using the confusion matrix [15]. Precision and recall are defined as:

$$
\begin{aligned}
& \text { Precision }=\frac{\mathrm{TP}}{\mathrm{TP}+\mathrm{FP}} \\
& \text { Recall }=\frac{\mathrm{TP}}{\mathrm{TP}+\mathrm{FN}}
\end{aligned}
$$

To a class in training dataset, TP (True Positive) denotes the number of correctly classified positive instances, FN (false negative) denotes the number of positive instances incorrectly classified as negative; TN (true negative) denotes the number of correctly classified negative instances and FP( false positive) denotes number of negative instances incorrectly classified as positive. The precise and recall of each class is shown in table 1.

Table.1 Confusion matrix of the decision tree

\begin{tabular}{|c|c|c|c|c|c|c|c|c|c|}
\hline & \multicolumn{4}{|c|}{ Classified as } & \multicolumn{5}{|c|}{ Measurement } \\
\hline Class & WLG & WUS & WDS & Other & TP & FP & FN & Precision & Recall \\
\hline WLG & 93 & 1 & 2 & 4 & 93 & 4 & 7 & $95.9 \%$ & $93.0 \%$ \\
\hline WUS & 1 & 87 & 0 & 4 & 87 & 8 & 5 & $91.6 \%$ & $94.6 \%$ \\
\hline WDS & 0 & 1 & 80 & 6 & 80 & 5 & 7 & $94.1 \%$ & $92.0 \%$ \\
\hline Other & 3 & 6 & 3 & 115 & 115 & 14 & 12 & $89.1 \%$ & $90.6 \%$ \\
\hline
\end{tabular}

\section{EXPERIMENT RESULT}

The decision tree based pedometer is tested in a walking experiment and an anti-interference experiment. Subjects were asked to wear a Samsung Gear fit [16], a wearable device, in the two experiments. Then the efficiency of the proposed system can be compared with that of the Gear fit. Four subjects participated in these two experiments. In the walking experience, each subject was asked to take 200 steps on level ground, go up 4 floors, then go down 4 floors. Each floor has 16 stairs. In the anti-interference experiment, subjects were asked to shake or swing the mobile phone and the Gear fit 10 times at the same time and to see whether the pedometer and Gear fit take those motions as steps. Samsung Gear fit cannot classify gait patterns. The accuracy of Gear fit in Table 1 only represents the accuracy of step detection. In the walking experiment, the overall classification accuracy is $89.4 \%$. In the anti-interference experiment, the average false steps recorded by the pedometer are 2.5 , while Gear fit produces 12 false steps, as shown in Table 2 .

Table .1 Accuracy of step detection

\begin{tabular}{|l|c|c|c|c|c|}
\hline & & \multicolumn{2}{|c|}{ Proposed Pedometer } & \multicolumn{2}{c|}{ Samsung Gear Fit } \\
\hline $\begin{array}{l}\text { Gait } \\
\text { Pattern }\end{array}$ & $\begin{array}{l}\text { Total } \\
\text { Steps }\end{array}$ & Steps Detected & Accuracy of Step Detection & $\begin{array}{l}\text { Steps } \\
\text { Detected }\end{array}$ & $\begin{array}{l}\text { Accuracy of } \\
\text { Step detection }\end{array}$ \\
\hline WLG & 800 & 776 & $97.0 \%$ & 779 & $97.4 \%$ \\
\hline WUS & 256 & 230 & $89.8 \%$ & 235 & $91.8 \%$ \\
\hline WDS & 256 & 238 & $92.9 \%$ & 210 & $82.0 \%$ \\
\hline Average & ------ & ----- & $93.2 \%$ & ----- & $90.4 \%$ \\
\hline
\end{tabular}


Table .2 Accuracy of classification

\begin{tabular}{|l|c|c|c|}
\hline \multicolumn{3}{|c|}{ Proposed Pedometer } \\
\hline Gait Pattern & Total Steps & Steps Correctly Classified & Accuracy of Classification \\
\hline WLG & 800 & 752 & $94.0 \%$ \\
\hline WUS & 256 & 218 & $85.2 \%$ \\
\hline WDS & 256 & 228 & $89.1 \%$ \\
\hline Average & ----- & ----- & $89.4 \%$ \\
\hline
\end{tabular}

Table 3. Result of anti-interference experiment

\begin{tabular}{|l|c|c|c|c|c|}
\hline & \multicolumn{5}{|c|}{ False Steps Recorded } \\
\hline & Subject1 & Subject2 & Subject3 & Subject4 & Average \\
\hline Proposed Pedometer & 2 & 0 & 4 & 4 & 2.5 \\
\hline Gear fit & 13 & 10 & 17 & 8 & 12 \\
\hline
\end{tabular}

\section{CONCLUSION}

A decision-based pedometer that can count steps and classify 3 gait patterns is developed. An angular velocity based algorithm is used in this pedometer to segment signals and enable the pedometer to count steps of different gait patterns easily. The decision tree is used to improve the accuracy and reliability of the pedometer. The system has been tested in several experiments with good results. The experiment results show that the proposed pedometer produces much less false step count than a commercial product.

\section{ACKNOWLEDGEMENTS}

This work is supported by City University of Hong Kong (Project 6987027).

\section{REFERENCES}

[1] E.P.Doheny, T.G.Foran and B.R.(2010) Greene, "A single gyroscope method for spatial gait analysis," in Proc. EMBC, pp. 1300-1303.

[2] Y.P.Lim, I.T.Brown and J.C.T.Khoo,( 2008) "An accurate and robust gyroscope-based pedometer," in Proc. EMBS, pp. 4587-4590.

[3] B.Aguiar, T.Rocha, J.Silva and I.Sousa,(2014) "Accelerometer-based fall detection for smartphones," in Proc. MeMeA, pp. 1-6.

[4] J.Mantyjarvi, J.Himberg and T.Seppanen,(2001) "Recognizing human motion with multiple acceleration sensors," in Proc. Systems, Man, and Cybernetics, vol. 2, pp.747-752.

[5] Rokach, L., O.Maimon,(2008) Data mining with decision trees: theory and applications, World Scientific Pub Co Inc, pp vii and 71.

[6] H.H.Manap, N.Md Tahir, R.Abdullah,(2013) "Parkinsonian Gait Motor Impairment Detection Using Decision Tree," in Proc. EMS, pp. 209-214.

[7] N.H.Lovell, N.Wang, E.Ambikairajah, B.G. Celler,(2007) "Accelerometry Based Classification of Walking Patterns Using Time-frequency Analysis," in Proc. EMBS, pp. 4899-4902.

[8] J.S.Wang, C.W.Lin, T.C.Yang, Y.J Ho,(2012) "Walking Pattern Classification and Walking Distance Estimation Algorithms Using Gait Phase Information," IEEE Trans. Biomedical Engineering, vol. 59, no. 10 , pp. 2884-2892.

[9] Motion Sensors in Android http://developer.android.com/guide/topics/sensors/sensors_motion.html

[10] R.O.Duda, P.E.Hart \& D. G. Stork,(2001) Pattern Classification. 2nded, New York: Wiley. 
[11] Z.Chi and H.Yan, (1996) "ID3-derived fuzzy rules and optimized defuzzification for handwritten numeral recognition," IEEE Trans. on Fuzzy Systems, vol. 4, no. 1, pp. 24-31.

[12] S.Zhao, Z.Chi, P. Shi and H. Yan, (2003) "Tow-stage segmentation of handwritten Chinese characters based on fuzzy decision rules," Pattern Recognition, vol. 36, pp. 145-156.

[13] A.Murad, T.Sarkodie-Gyan, H.Y.Yu, O.Fuentes, R.Brower. \& A.Abdelgawad, (2011) "Automatic classification of pathological gait patterns using ground reaction forces and machine learning algorithms," EMBC, pp.453-457.

[14] N.V.Chawla, K.W.Bowyer, L.O.Hall, and W.P.Kegelmeyer, (2002) "SMOTE: Synthetic Minority Over-sampling Technique", Journal of Artificial Intelligence Research, vol. 16, pp. 321 -357.

[15] D.L.Olson \& D.Delen (2008) Advanced Data Mining Techniques. Springer.

[16] Information on Samsung Gear Fit:

http://www.samsung.com/global microsite/gear/gearfit_features.html

\section{AUTHORS}

Juanying Lin received his master degree in Electronic and Information Engineering from City University of Hong Kong. He is currently a research assistant in the Department of Electronic Engineering at City University of Hong Kong. His research interests include digital image processing, motion pattern recognition and mobile computing.

Leanne Chan received her $\mathrm{PhD}$ degree in Biomedical Engineering from University of Southern California. She is currently Assistant Professor in the Department of Electronic Engineering at City University of Hong Kong. Her research interests include retinal prosthesis testbed development and microelectronic systems.

Hong Yan received his Ph.D. degree from Yale University. He was professor of imaging science at the University of Sydney and currently is professor of computer engineering at City University of Hong Kong. He was elected an IAPR fellow for contributions to document image analysis and an IEEE fellow for contributions to image recognition techniques and applications. His research interests include bioinformatics, image processing and pattern recognition.
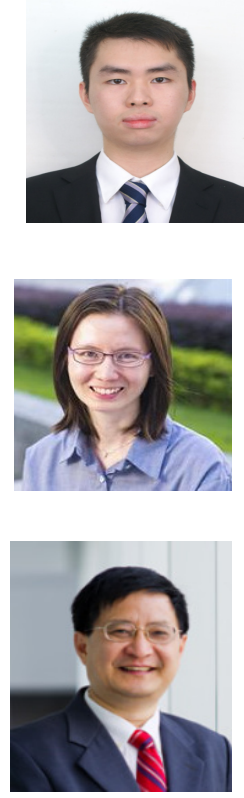